\title{
ON THE ANGULAR CORRELATION FUNCTIONS OF THE HUBBLE DEEP FIELD
}

\author{
BOUDEWIJN F. ROUKEMA \\ National Observatory of Japan, Mitaka, 181 Tokyo, Japan \\ email: roukema@iap.fr
}

Roukema \& Valls-Gabaud (1997) reinforce the conclusion of Colley et al. $(1996,1997)$ that the Hubble Deep Field (HDF) "galaxies" are probably star-forming regions, not "building-blocks".

Consider a "building-block" hypothesis:

(1) all (colour-selected high $z$ ) HDF galaxy-like objects are galaxies;

(2) these objects have a spatial correlation function $\xi(r, z)=b^{2}\left(r_{0} / r\right)^{\gamma}$ $(1+z)^{-(3+\epsilon-\gamma)}$ where $b \gg 1$ is a strong bias factor at high $z$ (e.g., Ogawa et al. 1997; see Groth \& Peebles 1977 and Roukema \& VallsGabaud 1997 for other parameters) and $b \geq 1, \partial b / \partial r<0 \forall r, z$; such that the projection of $\xi$ (3-D) into $w$ (angular correlation; 2-D), via Limber's equation (Limber 1953), matches Figs 1a,1d of Colley et al. (1996).

Since $w\left(1^{\prime \prime}\right) \gtrsim 1$ in Figs 1a,1d of Colley et al. (1996), at least $50 \%$ of the $1^{\prime \prime}$ object pairs can be considered "excess pairs". Table 1 of Roukema \& Valls-Gabaud (1997) therefores shows, conservatively, that of all the $1^{\prime \prime}$ object pairs, and under the above hypotheses, $25 \%$ are spatially separated by a median of only $3-7 h^{-1} \mathrm{kpc}$ (proper units), and $45 \%$ are spatially separated by a median of $12-30 h^{-1} \mathrm{kpc}^{1}$, taking into account projection effects. Many excess pairs have $\theta \sim 0.25$ ". Hence, for a pure "buildingblock" model, galaxy formation models (e.g., Roukema et al. 1998) would have to post-dict the existence of many $R_{\text {halo }} \ll 2 \mathrm{kpc}$ (proper units), very highly biased galaxies at $2.5 \lesssim z \lesssim 5$. This result is not very sensitive to $\epsilon$, $\Omega_{0}, \lambda_{0}$ or $z_{\text {median }}$ (Roukema \& Valls-Gabaud 1997).

\section{References}

Colley, W.N., Rhoads, J.E., Ostriker, J.P. \& Spergel, D.N. 1996, ApJ, 473, L63

Colley, W.N., Gnedin, O.Y., Ostriker, J.P. \& Rhoads, J.E. 1997, ApJ, 488, 579

Groth, E.J. \& Peebles, P.J.E. 1977, $A p J$, 217, 385

Limber, D.N. 1953, ApJ, 117, 134

Ogawa, T., Roukema, B.F., Yamashita, K. 1997, ApJ, 484, 53

Roukema, B.F., Peterson, B.A., Quinn, P.J. \& Rocca-Volmerange, B. 1998, MNRAS, in press (astro-ph/9707294)

Roukema, B.F. \& Valls-Gabaud, D. 1997, ApJ, 488, 524 